\title{
Modeling of Ash Clouds and Plumes Propagation during Explosive Eruptions of the Kamchatka Volcanoes
}

\author{
Sergey I. Malkovsky \\ Computing Center FEB RAS, Khabarovsk, Russia \\ sergey.malkovsky@gmail.com
}

\begin{abstract}
Ash clouds and plumes appearing during the explosive eruptions of Kamchatka volcanoes pose a danger to the public and economy, including air transportation and airports. For more than 30 years, scientists have carried out numerical modeling of ash clouds propagation to reduce the danger of air transportation in areas of volcanoes. The paper provides an overview of the main models of ash propagation used by the Volcanic Ash Advisory Centers. Examples are given of using of PUFF and FALL3D models for solving problems related to the operational forecast of ash clouds propagation during eruptions of the Kamchatka volcanoes. Methods considered for clarification parameters of volcanic eruptions using a joint analysis of the simulation results performed by means of the automated information system "Signal" and satellite data from the VolSatView information system.
\end{abstract}

Keywords: volcanic ash, numerical simulation, remote sensing, information system, VolSatView, AIS Signal, FALL3D, PUFF

Copyright (C2019 by the paper's authors. Copying permitted for private and academic purposes 


\title{
МОДЕЛИРОВАНИЕ РАСПРОСТРАНЕНИЯ ПЕПЛОВЫХ ОБЛАКОВ И ШЛЕЙФОВ ВО ВРЕМЯ ЭКСПЛОЗИВНЫХ ИЗВЕРЖЕНИЙ ВУЛКАНОВ КАМЧАТКИ
}

\author{
С.И. Мальковский \\ Вычислительный центр ДВО РАН, Хабаровск, Россия \\ sergey.malkovsky@gmail.com
}

\begin{abstract}
Пепловые облака и шлейфы, возникающие во время эксплозивных извержений вулканов Камчатки, представляют опасность для населения и народного хозяйства, в том числе для авиаперевозок и аэропортов. Более 30 лет ученые проводят численное моделирование распространения пепловых облаков для снижения опасности авиаполетов в районах вулканов. В работе приводится обзор основных моделей распространения пепла, используемых Консультационными центрами по вулканическому пеплу. Показаны примеры применения моделей PUFF и FALL3D для решения задач, связанных с оперативным прогнозом распространения пепловых облаков во время извержений вулканов Камчатки. Рассматриваются способы уточнения параметров эксплозивных извержений вулканов с помощью совместного анализа результатов моделирования, выполняемого средствами автоматизированной информационной системы “Сигнал”, и спутниковых данных из информационной системы VolSatView.
\end{abstract}

Ключевые слова: вулканический пепел, численное моделирование, спутниковый мониторинг, информационная система, VolSatView, АИС Сигнал, FALL3D, PUFF.

\section{Введение}

Пепловые облака и шлейфы, возникающие при эксплозивных извержениях вулканов, представляют большую опасность для воздушного транспорта. Облака, состоящие из частиц пепла размером менее 0,1 мм, могут оставаться в атмосфере продолжительное время, а на больших высотах переноситься ветром на тысячи километров от вулканов. Попадание самолета в пепловое облако может привести к отказу двигателей, абразивному истиранию оконных стекол и аэродинамических поверхностей, засорению и перегреву электроники и т. д. [1]. С распространением реактивных авиалайнеров во второй половине XX века появилась задача прогноза перемещения пепловых облаков и шлейфов, остающаяся актуальной до сих пор.

Одним из основных инструментов, используемых для решения этой задачи, является численное моделирование. Существует ряд факторов, влияющих на точность определения положения и характеристик пепловых облаков: погрешности в прогнозных метеорологических данных; погрешности, возникающие при определении начальных параметров пеплового облака; упрощенное описание эксплозивного процесса, а также процессов, происходящих в пепловом облаке при его распространении, заложенное в модели и т. д. Поэтому для повышения точности моделирования перемещения пепловых облаков требуется проводить анализ влияния начальных параметров используемой модели (включая пространственное и временное разрешение входных метеоданных) на полученные результаты, и сравнивать их с фактическими данными. Также необходимо разрабатывать новые методы определения как можно большего числа начальных 
параметров пеплового облака по данным различных наблюдений. Особенности географического положения вулканов Камчатки накладывают различные ограничения в применении наземных технических средств наблюдений и способствуют активному использованию для этих целей данных, получаемых методами дистанционного зондирования Земли.

В статье приводится обзор основных моделей расчета траектории распространения пепловых облаков и шлейфов, используемых Консультационными центрами по вулканическому пеплу (VAAC - Volcanic Ash Advisory Centers) Международной организации гражданской авиации (ICAO - International Civil Aviation Organization). Показаны примеры применения моделей PUFF [2] и FALL3D [3] для решения задач, связанных с оперативным прогнозом распространения пепловых облаков во время извержений вулканов Камчатки. Рассматриваются способы уточнения параметров эксплозивных извержений вулканов с помощью совместного анализа результатов моделирования, выполняемого средствами автоматизированной информационной системы (АИС) “Сигнал” [4], и спутниковых данных из информационной системы (ИС) VolSatView [5].

\section{Модели распространения пепловых облаков}

Среди всех моделей, используемых при моделировании распространения пепловых облаков, следует выделить модели, применяемые в VAAC. Данные центры были основаны в 1990-х годах международной организацией гражданской авиации с целью предоставления консультативной информации об объеме и перемещении вулканического пепла в атмосфере, представляющего опасность для авиасообщения. К основным моделям, используемым в данных центрах, относятся: CANERM [6], FALL3D, HYSPLIT [7], JMA [8], MLDPn [9], MOCAGE [10], NAME [11] и PUFF. Как и любые другие модели распространения примеси в атмосфере, указанные модели включают в себя следующие компоненты: модель источника примеси (высота облака, массовый расход вещества, вертикальное распределение массы и т. д.), модель распространения примеси (перенос, диффузия, сухое и влажное осаждение и т. д.), а также метеорологическую модель, являющуюся источником фактических или прогнозных данных о метеорологических полях (ветра, температуры и т. д.). При этом именно подход к описанию распространения примеси наиболее полно характеризует модели распространения пепла, которые по данному критерию могут быть подразделены на лагранжевы и эйлеровы [12].

Любая лагранжева модель основывается на идее о том, что частицы пепла перемещаются в атмосфере вдоль траекторий, определяемых полем ветра и эффектами турбулентности. Пепловое облако представляется в виде совокупности модельных частиц, каждая из которых имеет свое индивидуальное положение в пространстве, координаты которого рассчитываются по итерационной формуле на каждом временном шаге моделирования. Преимуществами данного класса моделей является высокая скорость их работы в оперативном режиме, когда требуется получить качественное представление о траектории движения облака. При необходимости определения количественных характеристик облака пепла (концентрация пепла в некоторой точке облака, объем пепла, выпавшего на поверхность, и т. д.) требуется значительное увеличение числа модельных частиц, что приводит к возрастанию вычислительной сложности модели [13]. В связи с этим модели данного типа имеют ограниченную применимость при моделировании распространения пепла в глобальных масштабах. К лагранжевым моделям относятся модели JMA, MLDPn, NAME и PUFF.

В отличие от лагранжевых, эйлеровы модели основываются на уравнении неразрывности для некоторого класса загрязняющего вещества, которое численно решается различными сеточными методами. Их основной областью применения является определение количественных характеристик облака пепла, в особенности при его перемещении на большие расстояния от вулкана. К этому классу моделей относятся модели: CANERM, FALL3D и MOCAGE. Попыткой совмещения сильных сторон рассмотренных двух классов моделей является применение при моделировании гибридного подхода, при котором на небольшом расстоянии от вулкана распространение облака пепла описывается с использованием лагранжева подхода, а при его 
удалении от вулкана применяется эйлеров подход. Примером такой модели является модель HYSPLIT.

Источником метеорологических данных, используемых при оперативном прогнозировании распространения пепловых облаков, являются численные модели прогноза погоды GFS (Global Forecast System), ECMWF IFS (Integrated Forecasting System), JMA GSM и т. д.

Сотрудниками Вычислительного центра (ВЦ) ДВО РАН и Института вулканологии и сейсмологии (ИВиС) ДВО РАН ведется исследование эффективности моделей распространения пепла, а также выполняется их адаптация для оперативного прогноза распространения пепловых облаков и шлейфов во время эксплозивных извержений вулканов Камчатки. В настоящее время используются модели PUFF и FALL3D совместно с прогностическими метеорологическими данными модели GFS. Такой выбор моделей обусловлен успешным опытом их длительного применения при изучении эксплозивных извержений вулканов в различных районах мира, и тем, что они имеют свободную программную реализацию, что позволяет дорабатывать модели при необходимости учета особенностей изучаемых опасных природных объектов.

В качестве примера на рис. 1 приведены результаты моделирования распространения пепла, полученные моделями PUFF и FALL3D с использованием прогностической информации модели GFS, для эксплозивного события вулкана Шивелуч 3 марта 2015 г. (VONA/KVERT WEEKLY INFORMATION RELEASE 10-2015, http://www.kscnet.ru/ivs/kvert/van/?n=10-2015) [1]. Исследуемое событие длительностью 80 минут началось 2015.03.03 в 22:50 UTC. Пепел поднялся на высоту 8-12 км. Указанные параметры, использованные при моделировании, были получены из сообщения No. 2015/73 VAAC Токио метеорологического агентства Японии (http://ds.data.jma.go.jp/svd/vaac/data/TextData/2015/20150303_SHEV_0073_Text.html), а также из сообщения Камчатского филиала Федерального исследовательского центра «Единая геофизическая служба РАН» $\quad$ от $3 \quad$ марта 2015 года (http://www.emsd.ru/ ssl/monitoring/arhiv/2015/Mar/03_Mar.htm).
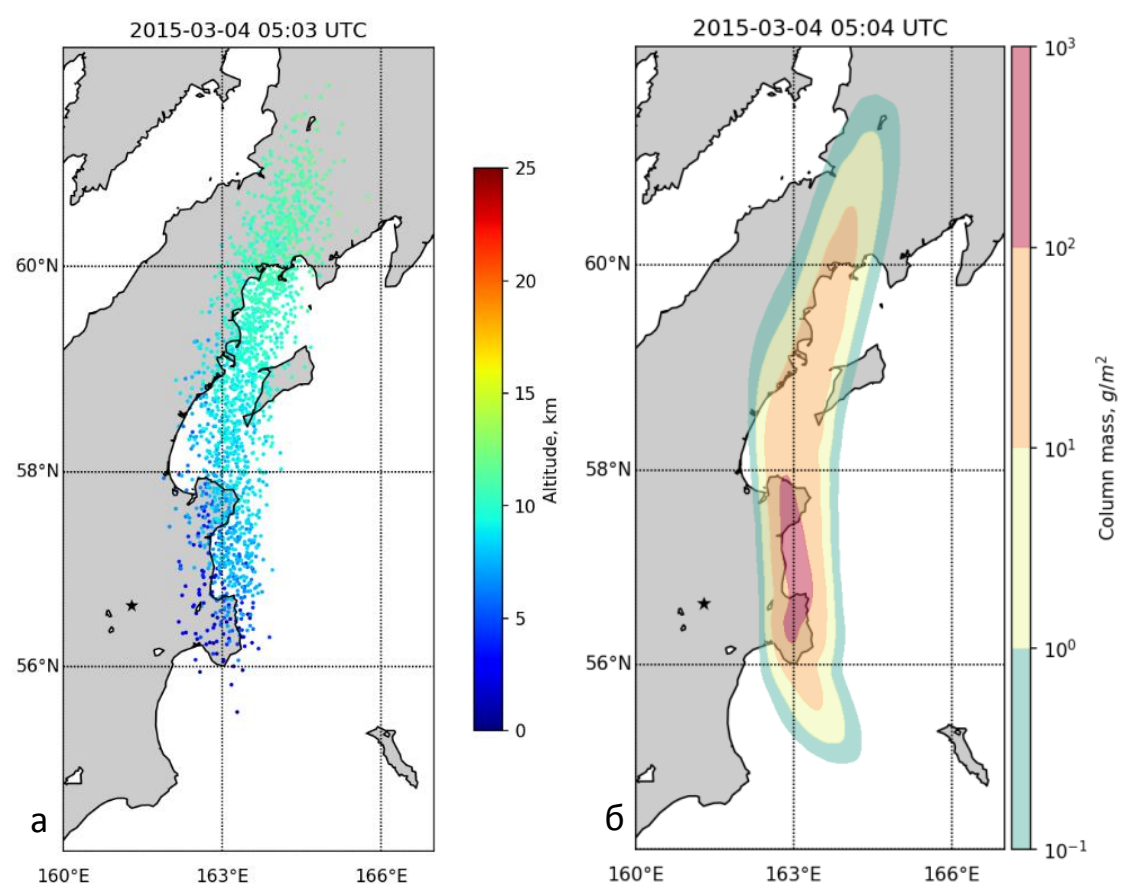

Рис. 1. Визуализаџия результатов расчетов распространения пепла во время эксплозивного события вулкана Шивелуч, выполненных с использованием моделей PUFF и FALL3D

Из рисунка видно, что положение пеплового облака, определенное по результатам расчетов с использованием модели PUFF (рис. 1a) и FALL3D (рис. 1б) практически совпадает. При этом модель PUFF позволяет определить высоту и направление перемещения облака пепла в 
оперативном режиме, а модель FALL3D - такие параметры облака, как концентрация пепла, масса пепла, выпавшего на поверхность и т. д.

\section{Совместный анализ результатов моделирования и спутниковых данных}

Большинство вулканов Камчатки находятся на значительном удалении от населенных пунктов и не покрыты достаточно плотной сетью наземных наблюдений. Поэтому постоянный мониторинг и анализ их активности невозможен без использования дистанционных, в первую очередь спутниковых, систем наблюдений. Для решения этих задач в 2011-2014 гг. сотрудниками ИВиС ДВО РАН, Института космических исследований (ИКИ) РАН, ВЦ ДВО РАН и Дальневосточного центра НИЦ "Планета" была создана информационная система VolSatView [14]. Она содержит архивы разнообразных спутниковых данных, а также широкий набор инструментов, предназначенных для работы с ними. Примеры использования ИС VolSatView для анализа активности вулканов Камчатки представлены в работах $[5,15]$.

В 2014-2015 гг. в ВЦ ДВО РАН по просьбе вулканологов ИВиС ДВО РАН была разработана подсистема, использующая модель PUFF, метеоданные модели GFS и разработанные программные средства в составе автоматизированной информационной системы (АИС) “Сигнал”, позволяющая выполнять моделирование распространения пепловых облаков и шлейфов при извержениях вулканов. Интеграция этой подсистемы с ИС VolSatView, а также реализация инструментов для совместного анализа результатов расчетов распространения пепла и спутниковых данных, значительно расширила возможности исследования эксплозивных извержений вулканов Камчатки и Курил. Так, совместный анализ данных, позволяет выполнять восстановление или уточнение некоторых параметров эксплозивных событий [16].

На рис. 2 показан пример варьирования начальной высоты пеплового облака эксплозивного извержения вулкана Ключевской, начавшегося 2017.08.15 в 20:00 UTC (http://www.kscnet.ru/ivs/kvert/van/?n=2017-197). Из рис. 2в видно, что оптимальное совпадение результатов моделирования со спутниковыми данными было получено при начальной высоте облака 7-8 км. При начальной высоте облака 0-7 км (рис. 2а) и 8-12 км (рис. 2б) пепловый шлейф лежит севернее и южнее контура, подготовленного на основе спутниковых данных, соответственно. Таким образом, совместный анализ результатов моделирования со спутниковыми данными позволил установить как начальную высоту пеплового облака, так и высоты дальнейшего распространения пепла.

Следует отметить, что подсистема моделирования, реализованная в составе АИС “Сигнал”, обладает рядом ограничений. Так она плохо подходит для прогноза распространения пепла на большие расстояния от вулкана. Также не поддерживается определение количественных характеристик пепловых облаков.

\section{Заключение}

Обзор различных моделей и анализ результатов их апробации для расчетов траектории распространения пепла показывает, что каждая из них имеет свои достоинства и недостатки. Поэтому при изучении эксплозивных событий необходимо использовать комплексный подход, заключающийся в анализе результатов сразу нескольких из них. Для решения этой задачи в АИС “Сигнал” будет дополнительно реализована подсистема моделирования, основанная на модели FALL3D. Это позволит перейти к работе с количественными характеристиками пепловых облаков, возникающих при эксплозивных извержениях вулканов Камчатки. 

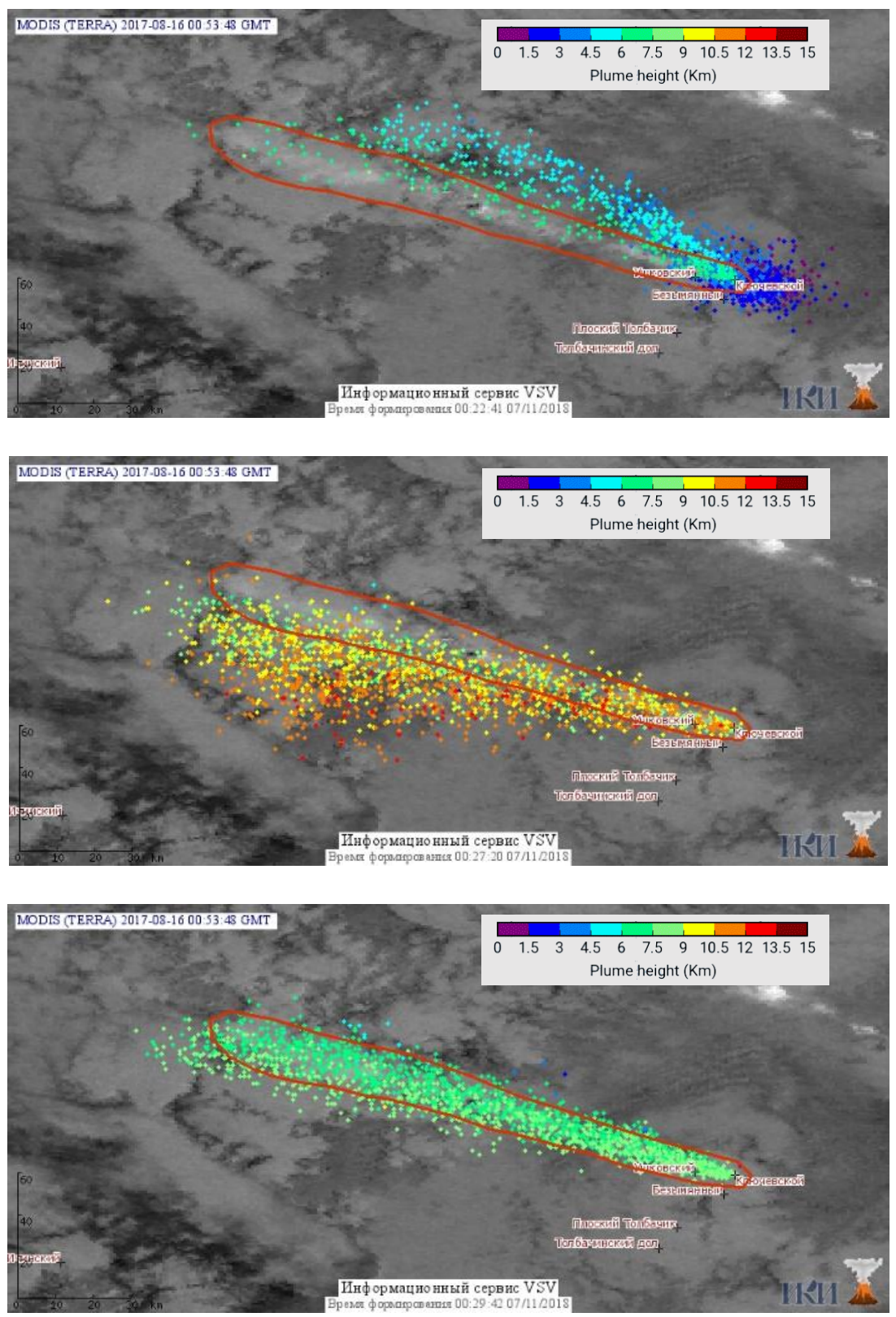

Рис. 2. Пример возможности варьирования начальной высоты пеплового облака, возникшего при эксплозивном извержении вулкана Ключевской 15 августа 2017 года в 20:00 UTC, для получения оптимального совпадения результатов моделирования и спутниковых данных. Черно-белое изображение - разница каналов 11-12 мкм по данным прибора MODIS (спутник Terra) на 00:53 UTC. Контур пеплового облака (красная линия) выполнен специалистом KVERT

Также, для повышения достоверности моделирования распространения вулканического пепла в АИС “Сигнал” будет обеспечена возможность использования региональных прогностических данных от численной модели прогноза погоды WRF-ARW. Из-за повышения требований к объему вычислений будет разработана параллельная реализация модели PUFF, что позволит проводить указанные расчеты в оперативном режиме.

Еще одним направлением развития АИС “Сигнал" является реализация методов усваивания результатов обработки спутниковых данных с их последующим использованием в моделях распространения пепла. Это необходимо для повышения точности прогноза распространения пепла и восстановления отдельных характеристик эксплозивных событий. В качестве спутниковых данных планируется использовать данные геостационарного спутника Himawari-8, обладающие высоким временным разрешением, достигающим 10 минут. 
Обработка данных выполнялась с использованием ресурсов Центров коллективного пользования “Центр данных ДВО РАН” (ВЦ ДВО РАН) [17] и “ИКИ-МониторинГ” (ИКИ РАН) [18]. При проведении расчетов применялись вычислительные технологии, разрабатываемые в рамках проекта РФФИ №. 18-29-03100.

\section{References}

[1] Gordeev E.I., Girina O.A., Volcanoes and their hazard to aviation, Herald of the Russian Academy of Sciences, 2014, 84(1), pp. 1-8. DOI: 10.1134/S1019331614010079.

[2] Searcy C., Dean K., Stringer W., PUFF: a high-resolution volcanic ash tracking model, Journal of Volcanology and Geothermal Research, 1998, 80(1-2), pp. 1-16.

[3] Costa A., Macedonio G., Folch A., A three-dimensional Eulerian model for transport and deposition of volcanic ashes, Earth and Planetary Science Letters, 2006, 241(3-4), pp. 634-647. DOI: 10.1016/j.epsl.2005.11.019.

[4] Sorokin A.A., Korolev S.P., Urmanov I.P., Verkhoturov A.L., Makogonov S.V., Shestakov N.V., Software Platform for Observation Networks Instrumental Data Far Eastern Branch of the Russian Academy of Sciences, Proceedings of International Conference on Computer Science and Environmental Engineering (CSEE 2015), Beijing, MAY 17-18, 2015, pp. 589-594. WOS:000361831900077.

[5] Gordeev E.I., Girina O.A., Lupyan E.A., Sorokin A.A., Kramareva L.S., Efremov V.Yu., Kashnitskii A.V., Uvarov I.A., Burtsev M.A., Romanova I.M., Mel'nikov D.V., Manevich A.G., Korolev S.P., Verkhoturov, A.L., The VolSatView information system for Monitoring the Volcanic Activity in Kamchatka and on the Kuril Islands, Journal of Volcanology and Seismology, 2016, 10(6), pp. 382-394. DOI: 10.1134/S074204631606004X.

[6] D'Amours R., Modeling the ETEX plume dispersion with the Canadian emergency response model, Atmospheric Environment, 1998, 32(24), pp. 4335-4341. DOI: 10.1016/S1352-2310(98)00182-4.

[7] Stein A.F., Draxler R.R., Rolph G.D., Stunder B.J.B., Cohen M.D., Ngan F., NOAA's HYSPLIT Atmospheric Transport and Dispersion Modeling System, Bulletin of the American Meteorological Society, 2015, 96(12), pp. 2059-2077. DOI: 10.1175/BAMS-D-14-00110.1.

[8] Iwasaki T., Maki T., Katayama K., Tracer transport model at Japan meteorological agency and its application to the ETEX data, Atmospheric Environment, 1998, 32(24), pp. 4285-4295. DOI: 10.1016/S13522310(98)00171-X.

[9] D’Amours R., Malo A., Flesch T., Wilson J., Gauthier J., Servranckx R., The Canadian Meteorological Centre's Atmospheric Transport and Dispersion Modelling Suite, Atmosphere-Ocean, 2015, 53(2), pp. 176-199. DOI: 10.1080/07055900.2014.1000260.

[10] Sič B., El Amraoui L., Marécal V., Josse B., Arteta J., Guth J., Joly M., Hamer P.D., Modelling of primary aerosols in the chemical transport model MOCAGE: Development and evaluation of aerosol physical parameterizations, Geoscientific Model Development, 2015, 8(2), pp. 381-408. DOI: 10.5194/gmd-8-381-2015.

[11] Ryall D.B., Maryon R.H., Validation of the UK Met. Offices name model against the ETEX dataset, Atmospheric Environment, 1998, 32(24), pp. 4265-4276. DOI: 10.1016/S1352-2310(98)00177-0.

[12] Belihov A.B., Legotin D.L., Suhov A.K., Sovremennye kompjuternye modeli rasprotraneniya zagryaznyauschih veschestv v atmosphere, Vestnik Kostromskogo Gosudarstvennogo Universiteta Im. N. A. Nekrasova, 2013, 1, pp. 14-19.

[13] Scollo S., Prestifilippo M., Coltelli M., Peterson R.A., Spata G., A statistical approach to evaluate the tephra deposit and ash concentration from PUFF model forecasts, Journal of Volcanology and Geothermal Research, 2011, 200(3-4), pp. 129-142. DOI: 10.1016/j.jvolgeores.2010.12.004. 
[14] Efremov V.Yu., Girina O.A., Kramareva L.S., Lupyan E.A., Manevich A.G., Mel'nikov D.V., Matveev A.M., Proshin A.A., Sorokin A.A., Flitman E.V., Creating an information service "Remote monitoring of active volcanoes of Kamchatka and the Kuril Islands", Sovremennye problemy distantsionnogo zondirovaniya Zemli iz kosmosa, 2012, 9(5), pp. 155-170.

[15] Gordeev E.I., Girina O.A., Lupyan E.A., Kashnitskiy A.V., Uvarov I.A., Efremov V.Yu., Melnikov D.V., Manevich A.G., Sorokin A.A., Verkhoturov A.L., Romanova I.M., Kramareva L.S., Korolev S.P., Studies of Kamchatka volcanic eruptions products using hyperspectral satellite data in VolSatView information system, Sovremennye problemy distantsionnogo zondirovaniya Zemli iz kosmosa, 2015, 12(1), pp. 113-128.

[16] Sorokin A.A., Girina O.A., Lupyan E.A., Mal'kovskii S.I., Balashov I.V., Efremov V.Y., Kramareva L.S., Korolev S.P., Romanova I.M., Simonenko E.V., Satellite observations and numerical simulation results for the comprehensive analysis of ash cloud transport during the explosive eruptions of Kamchatka volcanoes, Russian Meteorology and Hydrology, 2017, 42(12), pp. 759-765.

[17] Sorokin A.A., Makogonov S.I., Korolev S.P., The Information Infrastructure for Collective Scientific Work in the Far East of Russia, Scientific and Technical Information Processing, 2017, 4, pp. 302-304.

[18] Loupian E.A., Proshin A.A., Burtsev M.A., Balashov I.V., Bartalev S.A., Efremov V.Yu., Kashnitskii A.V., Mazurov A.A., Matveev A.M., Sudneva O.A., Sychugov I.G., Tolpin V.A., Uvarov I.A., IKI center for collective use of satellite data archiving, processing and analysis systems aimed at solving the problems of environmental study and monitoring, Sovremennye problemy distantsionnogo zondirovaniya Zemli iz kosmosa, 2015, 12(5), pp. 263-284. 